\title{
PlantCV v2: Image analysis software for high-throughput plant phenotyping
}

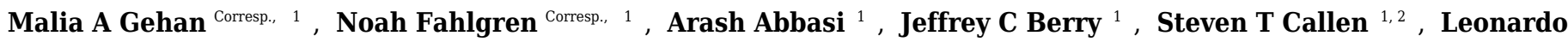
Chavez $^{1}{ }^{\text {， Andrew N Doust }}{ }^{3}$ ， Max J Feldman ${ }^{1}$ ， Kerrigan B Gilbert ${ }^{1}$ ， John G Hodge ${ }^{3}$ ， J Steen Hoyer ${ }^{1,4}$, Andy Lin ${ }^{1,5}$, Suxing Liu ${ }^{6,7}$, César Lizárraga ${ }^{1,8}$, Argelia Lorence ${ }^{9}$, Michael Miller ${ }^{1,10}$, Eric Platon ${ }^{11}$, Monica Tessman ${ }^{1,2}$, Tony Sax ${ }^{12}$

${ }^{1}$ Donald Danforth Plant Science Center, St. Louis, Missouri, United States of America

2 Current Address: Monsanto Company, St. Louis, Missouri, United States of America

3 Department of Plant Biology, Ecology, and Evolution, Oklahoma State University, Stillwater, Oklahoma, United States of America

${ }^{4}$ Computational and Systems Biology Program, Washington University in St. Louis, St. Louis, Missouri, United States of America

${ }^{5}$ Current Address: Unidev, St. Louis, Missouri, United States of America

${ }^{6}$ Arkansas Biosciences Institute, Arkansas State University, Jonesboro, Arkansas, United States of America

7 Current Address: Department of Plant Biology, University of Georgia, Athens, Georgia, United States of America

${ }^{8}$ Current Address: CiBO Technologies, Cambridge, Massachusetts, United States of America

${ }^{9}$ Arkansas Biosciences Institute, Department of Chemistry and Physics, Arkansas State University, Jonesboro, Arkansas, United States of America

${ }^{10}$ Current Address: Department of Agronomy and Horticulture, Center for Plant Science Innovation, Beadle Center for Biotechnology, University of Nebraska - Lincoln, Lincoln, Nebraska, United States of America

${ }^{11}$ Cosmos X, Tokyo, Japan

12 Missouri University of Science and Technology, Rolla, Missouri, United States of America

Corresponding Authors: Malia A Gehan, Noah Fahlgren

Email address: mgehan@danforthcenter.org, nfahlgren@danforthcenter.org

Systems for collecting image data in conjunction with computer vision techniques are a powerful tool for increasing the temporal resolution at which plant phenotypes can be measured non-destructively. Computational tools that are flexible and extendable are needed to address the diversity of plant phenotyping problems. We previously described the Plant Computer Vision (PlantCV) software package, which is an image processing toolkit for plant phenotyping analysis. The goal of the PlantCV project is to develop a set of modular, reusable, and repurposable tools for plant image analysis that are open-source and community-developed. Here we present the details and rationale for major developments in the second major release of PlantCV. In addition to overall improvements in the organization of the PlantCV project, new functionality includes a set of new image processing and normalization tools, support for analyzing images that include multiple plants, leaf segmentation, landmark identification tools for morphometrics, and modules for machine learning. 


\section{PlantCV v2: Image analysis software for high-throughput plant phenotyping}

2 Malia A Gehan*, 1, Noah Fahlgren*, 1, Arash Abbasi ${ }^{1}$, Jeffrey C Berry ${ }^{1}$, Steven T Callen ${ }^{1,2}$,

3 Leonardo Chavez ${ }^{1}$, Andrew N Doust ${ }^{3}$, Max J Feldman ${ }^{1}$, Kerrigan B Gilbert ${ }^{1}$, John G Hodge ${ }^{3}$, J

4 Steen Hoyer ${ }^{1,4}$, Andy Lin ${ }^{1,5}$, Suxing Liu ${ }^{6,7}$, César Lizárraga $^{1,8}$, Argelia Lorence ${ }^{9}$, Michael

5 Miller $^{1,10}$, Eric Platon ${ }^{11}$, Monica Tessman ${ }^{1,2}$, Tony Sax ${ }^{12}$

$6 \quad{ }^{1}$ Donald Danforth Plant Science Center, St. Louis, Missouri, United States of America

$7 \quad 2$ Current Address: Monsanto Company, St. Louis, Missouri, United States of America

$8{ }^{3}$ Department of Plant Biology, Ecology, and Evolution, Oklahoma State University, Stillwater,

9 Oklahoma, United States of America

$10{ }^{4}$ Computational and Systems Biology Program, Washington University in St. Louis, St. Louis,

11 Missouri, United States of America

$12{ }^{5}$ Current Address: Unidev, St. Louis, Missouri, United States of America

$13{ }^{6}$ Arkansas Biosciences Institute, Arkansas State University, Jonesboro, Arkansas, United States

14 of America

$15{ }^{7}$ Current Address: Department of Plant Biology, University of Georgia, Athens, Georgia, United

16 States of America

$17{ }^{8}$ Current Address: CiBO Technologies, Cambridge, Massachusetts, United States of America

$18{ }^{9}$ Arkansas Biosciences Institute, Department of Chemistry and Physics, Arkansas State

19 University, Jonesboro, Arkansas, United States of America

$20{ }^{10}$ Current Address: Department of Agronomy and Horticulture, Center for Plant Science

21 Innovation, Beadle Center for Biotechnology, University of Nebraska - Lincoln, Lincoln,

22 Nebraska, United States of America

$23{ }^{11}$ Cosmos X, Tokyo, Japan 
$24{ }^{12}$ Missouri University of Science and Technology, Rolla, Missouri, United States of America 25

$26 *$ These authors contributed equally to this work

27

28 Corresponding Author:

29 Malia Gehan; Noah Fahlgren

30975 North Warson Road, St. Louis, MO 63132, USA

31 Email address: mgehan@danforthcenter.org; nfahlgren@danforthcenter.org 


\section{ABSTRACT}

33 Systems for collecting image data in conjunction with computer vision techniques are a powerful

34 tool for increasing the temporal resolution at which plant phenotypes can be measured non-

35 destructively. Computational tools that are flexible and extendable are needed to address the

36 diversity of plant phenotyping problems. We previously described the Plant Computer Vision

37 (PlantCV) software package, which is an image processing toolkit for plant phenotyping

38 analysis. The goal of the PlantCV project is to develop a set of modular, reusable, and

39 repurposable tools for plant image analysis that are open-source and community-developed. Here

40 we present the details and rationale for major developments in the second major release of

41 PlantCV. In addition to overall improvements in the organization of the PlantCV project, new

42 functionality includes a set of new image processing and normalization tools, support for

43 analyzing images that include multiple plants, leaf segmentation, landmark identification tools

44 for morphometrics, and modules for machine learning. 


\section{INTRODUCTION}

46 All approaches for improving crops eventually require measurement of traits (phenotyping)

47 (Fahlgren, Gehan \& Baxter, 2015). However, manual plant measurements are time-consuming

48 and often require destruction of plant materials in the process, which prevents measurement of

49 traits for a single plant through time. Consequently, plant phenotyping is widely recognized as a

50 major bottleneck in crop improvement (Furbank \& Tester, 2011). Targeted plant phenotypes can

51 range from measurement of gene expression, to flowering time, to grain yield; therefore, the

52 software and hardware tools used are often diverse. Here, we focus on the software tools

53 required to nondestructively measure plant traits through images. This is a challenging area of

54 research because the visual definition of phenotypes vary depending on the target species. For

55 example, identification of petals can be used to measure flowering time, but petal color can vary

56 by species. Therefore, software tools needed to process high-throughput image data need to be

57 flexible and amenable to community input.

59 The term 'high-throughput' is relative to the difficulty to collect the measurement. The scale that

60 might be considered high-throughput for root phenotyping might not be the same for shoot

61 phenotyping, which can be technically easier to collect depending on the trait and species. Here

62 we define high-throughput as thousands or hundreds of thousands of images per dataset. PlantCV

63 is an open-source, open-development suite of analysis tools capable of analyzing high-

64 throughput image-based phenotyping data (Fahlgren et al., 2015). Version 1.0 of PlantCV

65 (PlantCV v1.0) was released in 2015 alongside the introduction of the Bellwether Phenotyping

66 Facility at the Donald Danforth Plant Science Center (Fahlgren et al., 2015). PlantCV v1.0 was

67 envisioned as a base suite of tools that the community could build upon, which lead to several 
68 design decisions aimed at encouraging participation. First, GitHub was used as a platform to

69 organize the community by integrating version control, code distribution, documentation, issue

70 tracking, and communication between users and contributors (Perez-Riverol et al., 2016).

71 Second, PlantCV was written in Python, a high-level language widely used for both teaching and

72 bioinformatics (Mangalam, 2002; Dudley \& Butte, 2009), to facilitate contribution from both

73 biologists and computer scientists. Additionally, the use of Python allows extension of PlantCV

74 with the many tools available from the Python scientific computing community (Oliphant, 2007;

75 Millman \& Aivazis, 2011). Third, a focus on modular development fosters code reuse and makes

76 it easier to integrate PlantCV with new or existing systems. Finally, the use of a permissive,

77 open-source license (MIT) allows PlantCV to be used, reused, or repurposed with limited

78 restrictions, for both academic and proprietary applications. The focus of the paper associated

79 with the original release of PlantCV v1.0 (Fahlgren et al., 2015) was not the structure and

80 function of PlantCV for image analysis, but rather an example of the type of biological question

81 that can be answered with high-throughput phenotyping hardware and software platforms. Since

82 the release of PlantCV v1.0 major improvements have been made to increase the flexibility,

83 usability, and functionality of PlantCV, while maintaining all of the functionality in v1.0. Here

84 we document the structure of PlantCV v2 along with examples that demonstrate new

85 functionality.

87 MATERIALS \& METHODS

88 The latest version or a specific release of PlantCV can be cloned from GitHub. The release for

89 this paper is v2.1. Scripts, notebooks, SQL schema, and simple input data associated with the

90 figures and results presented in this paper are available on GitHub at 
91 https://github.com/danforthcenter/plantcv-v2-paper. Project-specific GitHub repositories are kept

92 separate from the PlantCV software repository because their purpose is to make project-specific

93 analyses available for reproducibility, while the main PlantCV software repository contains

94 general purpose image analysis modules, utilities, and documentation.

95

96 Images of Arabidopsis thaliana were captured with a Raspberry Pi computer and camera in a

97 Conviron growth chamber. Additional details about the imaging set-up are provided in a

98 companion paper (Tovar et al., 2017). Images of Setaria viridis (A10) and Setaria italica (B100)

99 are from publicly available datasets that are available at

100 http://plantcv.danforthcenter.org/pages/data.html (Fahlgren et al., 2015; Feldman et al., 2017).

101 Images of wheat (Triticum aestivum L.) infected with wheat stem rust (Puccinia graminis f. sp.

102 tritici) were acquired with a flatbed scanner.

103

104 Image analysis was done in PlantCV using Python v2.7.5, OpenCV v2.4.5 (Bradski, 2000),

105 NumPy v1.12.1 (van der Walt, Colbert \& Varoquaux, 2011), Matplotlib v2.0.2 (Hunter, 2007),

106 SciPy v0.19.0 (Jones, Oliphant \& Peterson), Pandas v0.20.1 (McKinney \& Others, 2010), scikit-

107 image v0.13.0 (van der Walt et al., 2014), and Jupyter Notebook v4.2.1 (Kluyver et al., 2016).

108 Statistical analysis and data visualization was done using R v3.3 (R Core Team, 2017) and

109 RStudio v1.0 (RStudio Team, 2016). Graphs were produced using Matplotlib v2.0.2 (Hunter,

110 2007) and ggplot2 v2.2.1 (Wickham, 2009).

111 


\section{RESULTS AND DISCUSSION}

113 The following are details on improvements to the structure, usability, and functionality of

114 PlantCV since the v1.0 release. Further documentation for using PlantCV can be found at the

115 project website (http://plantcv.danforthcenter.org/).

116

\section{Organization of the PlantCV project}

118 PlantCV is a collection of modular Python functions, which are reusable units of Python code

119 with defined inputs and outputs (Fig. 1A). PlantCV functions can be assembled into simple

120 sequential or branching/merging pipelines. A pipeline can be as long or as short as it needs to be,

121 allowing for maximum flexibility for users using different imaging systems and analyzing

122 features of seed, shoot, root, or other plant systems. Suggestions on how to approach image

123 analysis with PlantCV, in addition to specific tutorials, are available through online

124 documentation (http://plantcv.readthedocs.io/en/latest/analysis approach/). Each function has a

125 debugging option to allow users to view and evaluate the output of a single step and adjust

126 parameters as necessary. A PlantCV pipeline is written by the user as a Python script. Once a

127 satisfactory pipeline script is developed, the PlantCV parallelization script ("plantcv-

128 pipeline.py') can be used to deploy the pipeline across a large set of image data (Fig. 1A). The

129 parallelization script also functions to manage data by consolidating measurements and metadata

130 into an SQLite database (Fig. 1B). In terms of speed, the user is only limited by the complexity

131 of the pipeline and the number of available processors.

132

133 The modular structure of the PlantCV package makes it easier for members of the community to

134 become contributors. Contributors to PlantCV submit bug reports, develop new functions and 
135 unit tests, or extend existing functionality or documentation. Core PlantCV developers do not

136 filter additions of new functions in terms of perceived impact or number of users but do check

137 that new functions follow the PlantCV contribution guide (see the sections on contributing in the

138 online documentation). PlantCV contributors are asked to follow the PEP8 Python style guide

139 (https://www.python.org/dev/peps/pep-0008/). Additions or revisions to the PlantCV code or

140 documentation are submitted for review using pull requests via GitHub. The pull request

141 mechanism is essential to protect against merge conflicts, which are sections of code that have

142 been edited by multiple users in potentially incompatible ways.

143

144 In PlantCV v2, several service integrations were added to automate common tasks during pull

145 requests and updates to the code repository. A continuous integration framework using the Travis

146 CI service (https://travis-ci.org/) was added so that software builds and unit tests can be run

147 automatically upon pull requests and other software updates. Continuous integration provides a

148 safeguard against code updates that break existing functionality by providing a report that shows

149 which tests passed or failed for each build (Wilson et al., 2014). The effectiveness of continuous

150 integration depends on having thorough unit test coverage of the PlantCV code base. Unit test

151 coverage of the PlantCV Python package is monitored through the Coveralls service

152 (https://coveralls.io/), which provides a report on which parts of the code are covered by existing

153 unit tests. In addition to the code, the PlantCV documentation was enhanced to use a continuous

154 documentation framework using the Read the Docs service (https://readthedocs.org/), which

155 allows documentation to be updated automatically and versioned in parallel with updates to

156 PlantCV. The documentation was updated to cover all functions in the PlantCV library, tutorials

157 on building pipelines and using specialized tools (e.g. multi-plant analysis and machine learning 
158 tools), a frequently asked questions section, and several guides such as installation, Jupyter

159 notebooks, and instructions for contributors.

160

161 Improved usability

162 PlantCV v1.0 required pipeline development to be done using the command line, where debug

163 mode is used to write intermediate image files to disk for each step. In command-line mode, an

164 entire pipeline script must be executed, even if only a single step is being evaluated. To improve

165 the pipeline and function development process in PlantCV v2, the debugging system was

166 updated to allow for seamless integration with the Juptyer Notebook system (http://jupyter.org/;

167 Kluyver et al., 2016). Jupyter compatibility allows users to immediately visualize output and to

168 iteratively rerun single steps in a multi-step PlantCV pipeline, which makes parameters like

169 thresholds or regions of interest much easier to adjust. Once a pipeline is developed in Jupyter, it

170 can then be converted into a Python script that is compatible with PlantCV parallelization (see

171 online documentation for detailed instructions on conversion;

172 http://plantcv.readthedocs.io/en/latest/jupyter/). Because of the web-based interface and useful

173 export options, Jupyter notebooks are also a convenient method of sharing pipelines with

174 collaborators, or in publications, and teaching others to use PlantCV.

175

176 PlantCV was initially created to analyze data generated by the Bellwether Phenotyping Facility

177 at the Donald Danforth Plant Science Center. Several updates to PlantCV v2 addressed the need

178 to increase the flexibility of PlantCV to analyze data from other plant phenotyping systems. The

179 PlantCV SQLite database schema was simplified so that new tables do not need to be added for

180 every new camera system (Fig. 1B). The full database schema is available on GitHub (see 
181 Materials and Methods) and in PlantCV documentation. New utilities were added to PlantCV v2

182 that allow data to be quickly and efficiently exported from the SQLite database into text files that

183 are compatible with R (R Core Team, 2017) for further statistical analysis and data visualization.

185 Because standards for data collection and management for plant phenotyping data are still being

186 developed (Pauli et al., 2016), image metadata is often stored in a variety of formats on different

187 systems. A common approach is to include metadata within image filenames, but because there

188 is a lack of file naming standards, it can be difficult to robustly capture this data automatically. In

189 PlantCV v2, a new metadata processing system was added to allow for flexibility in file naming

190 both within and between experiments and systems. The PlantCV metadata processing system is

191 part of the parallelization tool and works by using a user-provided template to process filenames.

192 User-provided templates are built using a restricted vocabulary so that metadata can be collected

193 in a standardized way. The vocabulary used can be easily updated to accommodate future

194 community standards.

195

\section{Performance}

197 In PlantCV v1.0, image analysis parallelization was achieved using a Perl-based multi-threading

198 system that was not thread-safe, which occasionally resulted in issues with data output that had

199 to be manually corrected. Additionally, the use of the Python package Matplotlib (Hunter, 2007)

200 in PlantCV v1.0 limited the number of usable processors to 10-12. For PlantCV v2, the

201 parallelization framework was completely rewritten in Python using a multiprocessing

202 framework, and the use of Matplotlib was updated to mitigate the issues and processor

203 constraints in v1.0. The output of image files mainly used to assess image segmentation quality 
204 is now optional, which should generally increase computing performance. Furthermore, to

205 decentralize the computational resources needed for parallel processing and prepare for future

206 integration with high-throughput computing resources that use file-in-file-out operations, results

207 from PlantCV pipeline scripts (one per image) are now written out to temporary files that are 208 aggregated by the parallelization tool after all image processing is complete.

210 New Functionality

211 PlantCV v2 has added new functions for image white balancing, auto-thresholding, size marker

212 normalization, multi-plant detection, combined image processing, watershed segmentation,

213 landmarking, and a trainable naive Bayes classifier for image segmentation (machine learning).

214 The following are short descriptions and sample applications of new PlantCV functions.

215

216 White balancing

217 If images are captured in a greenhouse, growth chamber, or other situation where light intensity

218 is variable, image segmentation based on global thresholding of image intensity values can

219 become variable. To help mitigate image inconsistencies that might impair the ability to use a

220 single global threshold and thus a single pipeline over a set of images, a white balance function

221 was developed. If a white color standard is visible within the image, the user can specify a region

222 of interest. If a specific area is not selected then the whole image is used. Each channel of the

223 image is scaled relative to the reference maximum. 
Auto-thresholding functions

226 An alternative approach to using a fixed, global threshold for image segmentation is to use an

227 auto-thresholding technique that either automatically selects an optimal global threshold value or

228 introduces a variable threshold for different regions in an image. Triangle, Otsu, mean, and

229 Gaussian auto-thresholding functions were added to PlantCV to further improve object detection

230 when image light sources are variable. The 'triangle_auto_threshold' function implements the

231 method developed by Zack et al. 1977 (Zack, Rogers \& Latp, 1977). The triangle threshold

232 method uses the histogram of pixel intensities to differentiate the target object (plant) from

233 background by generating a line from the peak pixel intensity (Duarte, 2015) to the last pixel

234 value and then finding the point (i.e., the threshold value) on the histogram that maximizes

235 distance to that line. In addition to producing the thresholded image in debug mode, the

236 'triangle_auto_threshold' function outputs the calculated threshold value and the histogram of

237 pixel intensities that was used to calculate the threshold. In cases where the auto-threshold value 238 does not adequately separate the target object from background, the threshold can be adjusted by

239 modifying the stepwise input. Modifying the stepwise input shifts the distance calculation along

240 the x-axis, which subsequently calculates a new threshold value to use.

242 The Otsu, mean, and Gaussian threshold functions in PlantCV are implemented using the

243 OpenCV library (Bradski, 2000). Otsu's binarization ['otsu_auto_threshold;' (Otsu, 1979)] is

244 best implemented when a grayscale image histogram has two peaks since the Otsu method

245 selects a threshold value that minimizes the weighted within-class variance. In other words, the

246 Otsu method identifies the value between two peaks where the variances of both classes are

247 minimized. Mean and Gaussian thresholding are executed by indicating the desired threshold 
248 type in the function 'adaptive_threshold.' The mean and Gaussian methods will produce a

249 variable local threshold where the threshold value of a pixel location depends on the intensities

250 of neighboring pixels. For mean adaptive thresholding, the threshold of a pixel location is

251 calculated by the mean of surrounding pixel values; for Gaussian adaptive thresholding, the

252 threshold value of a pixel is the weighted sum of neighborhood values using a Gaussian window

253 (Gonzalez \& Woods, 2002; Kaehler \& Bradski, 2016).

255 Gaussian blur

256 In addition to the 'median_blur' function included in PlantCV v1.0, we have added a Gaussian

257 blur smoothing function to reduce image noise and detail. Both the median and Gaussian blur

258 methods are implemented using the OpenCV library (Bradski, 2000) and are typically used to

259 smooth a grayscale image or a binary image that has been previously thresholded. Image

260 blurring, while reducing detail, can help remove or reduce signal from background noise (e.g.

261 edges in imaging cabinets), generally with minimal impact on larger structures of interest.

262 Utilizing a rectangular neighborhood around a center pixel, 'median_blur' replaces each pixel in

263 the neighborhood with the median value. Alternatively, 'gaussian_blur' determines the value of

264 the central pixel by multiplying its and neighboring pixel values by a normalized kernel and then

265 averaging these weighted values (i.e., image convolution) (Kaehler \& Bradski, 2016). The extent

266 of image blurring can be modified by increasing (for greater blur) or decreasing the kernel size

267 (which takes only odd numbers; commonly, 3x3) or by changing the standard deviation in the $\mathrm{X}$ 268 and/or Y directions. 
271 Images that are not collected from a consistent vantage point require one or more size markers as

272 references for absolute or relative scale. The size marker function allows users to either detect a

273 size marker within a user-defined region of interest or to select a specific region of interest to use

274 as the size marker. The pixel area of the marker is returned as a value that can be used to

275 normalize measurements to the same scale. For this module to function correctly we assume that

276 the size marker stays in frame, is unobstructed, and is relatively consistent in position throughout

277 a dataset, though some movement is allowed as long as the marker remains within the defined

278 marker region of interest.

279

280 Multi-plant detection

281 There is growing interest among the PlantCV user community to process images with multiple

282 plants grown in flats or trays, but PlantCV v1.0 was built to processes images containing single

283 plants. The major challenge with analyzing multiple plants in an image is successfully

284 identifying individual whole plants as distinct objects. Leaves or other plant parts can sometimes

285 be detected as distinct contours from the rest of the plant and need to be grouped with other

286 contours from the same plant to correctly form a single plant/target object. While creating

287 multiple regions of interest (ROI) to demarcate each area containing an individual plant/target is

288 an option, we developed two modules, 'cluster_contours' and 'cluster_contours_split_img,' that

289 allow contours to be clustered and then parsed into multiple images without having to manually

290 create multiple ROIs (Fig. 2).

291 
292 The 'cluster_contours' function takes as input: an image, the contours that need to be clustered, a

293 number of rows, and a number of columns. Total image size is detected, and the rows and

294 columns create a grid to serve as approximate ROIs to cluster the contours (Fig. 2A). The

295 number of rows and columns approximate the desired size of the grid cells. There does not need

296 to be an object in each of the grid cells. Several functions were also added to aid the clustering

297 function. The 'rotate_img' and 'shift_img' functions allow the image to be adjusted so objects

298 are better aligned to a grid pattern.

299

300 After objects are clustered, the 'cluster_contour_split_img' function splits images into the

301 individual grid cells and outputs each as a new image so that there is a single clustered object per

302 image. If there is no clustered object in a grid cell, no image is outputted. With the

303 'cluster_contour_split_img' function, a text file with genotype names can be included to add

304 them to image names. The 'cluster_contour_split_img' function also checks that there are the

305 same number of names as objects. If there is a conflict in the number of names and objects, a

306 warning is printed and a correction is attempted. Alternatively, if the file option is not used, all of

307 the object groups are labeled by position. Once images are split, they can be processed like single

308 plant images using additional PlantCV tools (Fig. 2B). See the online documentation for an

309 example multi-plant imaging pipeline (http://plantcv.readthedocs.io/en/latest/multi-

310 plant tutorial/).

311

312 The current method for multi-plant identification in PlantCV is flexible but relies on a grid

313 arrangement of plants, which is common for controlled-environment-grown plants. Future

314 releases of PlantCV may incorporate additional strategies for detection and identification of 
315 plants, such as arrangement-independent $K$-means clustering approaches (Minervini,

316 Abdelsamea \& Tsaftaris, 2014).

317

318 Combined image processing

319 The Bellwether Phenotyping Facility has both RGB visible light (VIS) and near-infrared (NIR)

320 cameras, and images are captured $\sim 1$ minute apart (Fahlgren et al., 2015). Compared to VIS

321 images, NIR images are grayscale with much less contrast between object and background. It can

322 be difficult to segment plant material from NIR images directly, even with edge detection steps.

323 Therefore, several functions were added to allow the plant binary mask that results from VIS

324 image processing pipelines to be resized and used as a mask for NIR images. Combining VIS

325 and NIR camera pipelines also has the added benefit of decreasing the number of steps necessary

326 to process images from both camera types, thus increasing image processing throughput. The

327 'get_nir' function identifies the path of the NIR image that matches VIS image. The 'get_nir'

328 function requires that the image naming scheme is consistent and that the matching image is in

329 the same image directory. The 'resize' function then resizes the VIS plant mask in both the $\mathrm{x}$ and

330 y directions to match the size of the NIR image. Resizing values are determined by measuring

331 the same reference object in an example image taken from both VIS and NIR cameras (for

332 example the width of the pot or pot carrier in each image). The 'crop_position_mask' function is

333 then used to adjust the placement of the VIS mask over the NIR image and to crop/adjust the VIS

334 mask so it is the same size as the NIR image. It is assumed that the pot position changes

335 consistently between VIS and NIR image datasets. An example VIS/NIR dual pipeline to follow

336 can be accessed online (http://plantcv.readthedocs.io/en/latest/vis_nir_tutorial/). 
339 While segmentation and analysis of whole plants in images provides useful information about

340 plant size and growth, a more detailed understanding of plant growth and development can be

341 obtained by measuring individual plant organs. However, fully automated segmentation of

342 individual organs such as leaves remains a challenge, due to issues such as occlusion (Scharr et

343 al., 2016). Multiple methods for leaf segmentation have been proposed (Scharr et al., 2016), and

344 in PlantCV v2 we have implemented a watershed segmentation approach. The

345 'watershed_segmentation' function can be used to estimate the number of leaves for plants

346 where leaves are distinctly separate from other plant structures (e.g. A. thaliana leaves are

347 separated by thin petioles; Fig. 3). The inputs required are an image, an object mask, and a

348 minimum distance to separate object peaks. The function uses the input mask to calculate a

349 Euclidean distance map (Liberti et al., 2014). Marker peaks calculated from the distance map

350 that meet the minimum distance setting are used in a watershed segmentation algorithm (van der

351 Walt et al., 2014) to segment and count the objects. Segmented objects are visualized in different

352 colors, and the number of segmented objects is reported (Fig. 3). An example of how the

353 watershed segmentation method was used to assess the effect of water deficit stress on the

354 number of leaves of $A$. thaliana plants can be found in Acosta-Gamboa et al. 2017 (Acosta-

355 Gamboa et al., 2017).

356

357 Landmarking functions for morphometrics

358 To extend PlantCV beyond quantification of size-based morphometric features, we developed

359 several landmarking functions. Landmarks are generally geometric points located along the

360 contours of a shape that correspond to homologous biological features that can be compared 
361 between subjects (Bookstein, 1991). Typical examples of landmarks include eyes between

362 human subjects or suture joins in a skull. For a growing plant, potential landmarks include the

363 tips of leaves and pedicel and branch angles. When specified a priori, landmarks should be

364 assigned to provide adequate coverage of the shape morphology across a single dimensional

365 plane (Bookstein, 1991). Additionally, the identification of landmark points should be repeatable

366 and reliable across subjects while not altering their topological positions relative to other

367 landmark positions (Bookstein, 1991). Type I landmarks provide the strongest support for

368 homology because they are defined by underlying biological features, but it is problematic to

369 assign Type I landmarks a priori when analyzing high-throughput plant imagery. To address

370 this, PlantCV v2 contains functions to identify anatomical landmarks based upon the

371 mathematical properties of object contours (Type II) and non-anatomical pseudo-

372 landmarks/semilandmarks (Type III), as well as functions to rescale and analyze biologically

373 relevant shape properties (Bookstein, 1991, 1997; Gunz, Mitteroecker \& Bookstein, 2005; Gunz

$374 \&$ Mitteroecker, 2013).

375

376 The 'acute' function identifies Type II landmarks by implementing a pseudo-landmark

377 identification algorithm that operates using a modified form of chain coding (Freeman, 1961).

378 Unlike standard chain coding methods that attempt to capture the absolute shape of a contour, the

379 acute method operates by measuring the angle between a pixel coordinate and two neighboring

380 pixels on opposite sides of it that fall within a set distance, or window, along the length of the

381 contour. The two neighboring points are used to calculate an angle score for the center pixel.

382 When the angle score is calculated for each position along the length of a contour, clusters of

383 acute points can be identified, which can be segmented out by applying an angle threshold. The 
384 middle position within each cluster of acute points is then identified for use as a pseudo-

385 landmark (Fig. 4A). The ability to subjectively adjust the window size used for generating angle

386 scores also helps to tailor analyses for identifying points of interest that may differ in resolution.

387 For example, an analysis of leaf data might utilize a larger window size to identify the tips of

388 lobes whereas smaller window sizes would be able to capture more minute patterns such as

389 individual leaf serrations. Further segmentation can also be done using the average pixel values

390 output (pt_vals) for each pseudo-landmark, which estimates the mean pixel intensity within the

391 convex hull of each acute region based on the binary mask used in the analysis. The average

392 pixel value output allows for concave landmarks (e.g. leaf axils and grass ligules) and convex

393 landmarks (e.g. leaf tips and apices) on a contour to be differentiated in downstream analyses.

394 Additionally, PlantCV v2 includes the 'acute_vertex' function that uses the same chain code-

395 based pseudo-landmark identification algorithm used in the 'acute' function except that it uses an

396 adjustable local search space criteria to reduce the number of angle calculations, which speeds up

397 landmark identification.

398

399 For Type III landmarks, the 'x_axis_pseudolandmarks' and ' $y \_$_axis_pseudolandmarks' functions

400 identify homologous points along a single dimension of an object (x-axis or y-axis) based on

401 equidistant point locations within an object contour. The plant object is divided up into twenty

402 equidistant bins, and the minimum and maximum extent of the object along the axis and the

403 centroid of the object within each bin is calculated. These sixty points located along each axis

404 possess the properties of semi/pseudo-landmark points (an equal number of reference points that

405 are approximately geometrically homologous between subjects to be compared) that approximate

406 the contour and shape of the object (Fig. 4B). Such semi/pseudo-landmarking strategies have 
407 been utilized in cases where traditional homologous landmark points are difficult to assign or

408 poorly represent the features of object shape (Bookstein, 1997; Gunz, Mitteroecker \& Bookstein,

409 2005; Gunz \& Mitteroecker, 2013).

410

411 Frequently, comparison of shape attributes requires rescaling of landmark points to eliminate the

412 influence of size on the relative position of landmark points. The landmark functions in PlantCV

413 output untransformed point values that can either be directly input into morphometric programs

414 in R [shapes (Dryden \& Mardia, 2016) or morpho (Schlager, Jefferis \& Schlager, 2016)] or

415 uniformly rescaled to a 0-1 coordinate system using the PlantCV 'scale_features' function. The

416 location of landmark points can be used to examine multidimensional growth curves for a broad

417 variety of study systems and tissue types and can be used to compare properties of plant shape

418 throughout development or in response to differences in plant growth environment. An example

419 of one such application is the 'landmark_reference_pt_dist' function. This function estimates the

420 vertical, horizontal, Euclidean distance, and angle of landmark points from two landmarks

421 (centroid of the plant object and centroid localized to the base of the plant). Preliminary evidence

422 from a water limitation experiment performed using a Setaria recombinant inbred population

423 indicates that vertical distance from rescaled leaf tip points identified by the 'acute_vertex'

424 function to the centroid is decreased in response to water limitation and thus may provide a

425 proximity measurement of plant turgor pressure (Fig. 4C and 4D).

426

427 Two-class or multiclass naive Bayes classifier

428 Pixel-level segmentation of images into two or more classes is not always straightforward using

429 traditional image processing techniques. For example, two classes of features in an image may be 
430 visually distinct but similar enough in color that simple thresholding is not sufficient to separate

431 the two groups. Furthermore, even with methods that adjust for inconsistencies between images

432 (e.g. white balancing and auto-thresholding functions), inconsistent lighting conditions in a

433 growth chamber, greenhouse, or field can still make bulk processing of images with a single

434 workflow difficult. Methods that utilize machine learning techniques are a promising approach to

435 tackle these and other phenotyping challenges (Minervini, Abdelsamea \& Tsaftaris, 2014; Singh

436 et al., 2016; Ubbens \& Stavness, 2017; Atkinson et al., 2017; Pound et al., 2017). With PlantCV

437 v2, we have started to integrate machine learning methods to detect features of interest (e.g. the

438 plant), starting with a naive Bayes classifier (Abbasi \& Fahlgren, 2016). The naive Bayes

439 classifier can be trained using two different approaches for two-class or multiclass (two or more)

440 segmentation problems. During the training phase using the 'plantcv-train.py' script, pixel RGB

441 values for each input class are converted to the hue, saturation and value (HSV) color space.

442 Kernel density estimation (KDE) is used to calculate a probability density function (PDF) from a

443 vector of values for each HSV channel from each class. The output PDFs are used to

444 parameterize the naive Bayes classifier function ('naive_bayes_classifier'), which can be used to 445 replace the thresholding steps in a PlantCV pipeline. The 'naive_bayes_classifer' function uses

446 these PDFs to calculate the probability (using Bayes' theorem) that a given pixel is in each class.

447 The output of the 'naive_bayes_classifier' is a binary image for each class where the pixels are

448 white if the probability the pixel was in the given class was highest of all classes and is black

449 otherwise. A tutorial of how to implement naive Bayes plant detection into an image processing

450 pipeline is online (http://plantcv.readthedocs.io/en/latest/machine_learning_tutorial/). 
452 For the two-class approach, the training dataset includes color images and corresponding binary

453 masks where the background is black and the foreground (plant or other target object) is white.

454 PlantCV can be used to generate binary masks for the training set using the standard image

455 processing methods and the new 'output_mask' function. It is important for the training dataset

456 to be representative of the larger dataset. For example, if there are large fluctuations in light

457 intensity throughout the day or plant color throughout the experiment, the training dataset should

458 try to cover the range of variation. A random sample of $10 \%$ of the foreground pixels and the

459 same number background pixels are used to build the PDFs.

460

461 To assess how well the two-class naive Bayes method identifies plant material in comparison to

462 thresholding methods, we reanalyzed Setaria images (Fahlgren et al., 2015) using the naive

463 Bayes classifier and compared the pixel area output to pipelines that utilize thresholding steps

464 (Fig. 5). We used 99 training images (14 top view and 85 side view images) from a total of 6473

465 images. We found that the plant pixel area calculated by naive Bayes was highly correlated with

466 that calculated from pipelines that use thresholding for both side-view images $\left(\mathrm{R}^{2}=0.99\right.$; Fig. 5A)

467 and top-view images $\left(\mathrm{R}^{2}=0.96\right.$; Fig. 5B). Naive Bayes segmentation enabled use of pipelines that

468 were both simpler (fewer steps) and more flexible: five new scripts were sufficient for processing

469 the dataset (five categories of photo data), whereas nine threshold-based pipeline scripts had

470 previously been required.

471

472 The multiclass naive Bayes approach requires a tab-delimited table for training where each

473 column is a class (minimum two) and each cell is a comma-separated list of RGB pixel values

474 from the column class. We currently use the Pixel Inspection Tool in ImageJ (Schneider, 
475 Rasband \& Eliceiri, 2012) to collect samples of pixel RGB values used to generate the training

476 text file. As noted above for the two-class approach, it is important to adequately capture the

477 variation in the image dataset for each class when generating the training text file to improve

478 pixel classification. If images are consistent, only one image needs to be sampled for generating

479 the training table; however, if they vary, several images may be needed. For complex

480 backgrounds (or non-targeted objects), several classes may be required to capture all of the

481 variation. Once the training table is generated, it is input into the 'plantcv-train.py' script to

482 generate PDFs for each class. As an example, we used images of wheat leaves infected with

483 wheat rust to collect pixel samples from four classes: non-plant background, unaffected leaf

484 tissue, rust pustule, and chlorotic leaf tissue, and then used the naive Bayes classifier to segment

485 the images into each class simultaneously (Fig. 6). This method can likely be used for a variety

486 of applications, such as identifying a plant under variable lighting conditions or quantifying

487 specific areas of stress on a plant.

488

489 In summary, the naive Bayes classifier offers several advantages over threshold-based

490 segmentation: 1) two or more classes can be segmented simultaneously; 2) probabilistic

491 segmentation can be more robust across images than fixed thresholds; and 3) classifier-based

492 segmentation replaces multiple steps in threshold-based pipelines, reducing pipeline complexity.

\section{CONCLUSIONS}

495 The field of digital plant phenotyping is at an exciting stage of development where it is

496 beginning to shift from a bottleneck to one that will have a positive impact on plant research,

497 especially in agriculture. The Plant Image Analysis database currently lists over 150 tools that 
498

499

500

501

502

503

504

505

506

507

508

509

510

511 There are several areas where we envision future PlantCV development. Standards and

512 interoperability: Improved interoperability of PlantCV with data providers and downstream

513 analysis tools will require adoption of community-based standards for data and metadata [e.g.

514 Minimum Information About a Plant Phenotyping Experiment; (Ćwiek-Kupczyńska et al.,

515 2016)]. Improved interoperability will make it easier to develop standardized tools for statistical 516

517 projects. New data sources: Handling and analysis of data from specialized cameras that

518 measure three-dimensional structure or hyperspectral reflectance will require development or

519 integration of additional methods into PlantCV. Machine learning: Our goal is to develop

520 additional tools for machine learning and collection of training data. In some cases, where these 
521 methods can be implemented in a modular and reusable framework, they can be integrated

522 directly into PlantCV. In other cases, PlantCV can be combined with new and existing tools. A

523 recent example of this latter approach built on PlantCV, using its image preprocessing and

524 segmentation functions alongside a modular framework for building convolutional neural

525 networks (Ubbens \& Stavness, 2017). As noted throughout, we see great potential for modular

526 tools such as PlantCV and we welcome community feedback.

527

\section{ACKNOWLEDGMENTS}

529 We would like to thank Melinda Darnell, Leonardo Chavez, Kevin Reilly, and the staff of both

530 the Danforth Center Facilities and Support Services group and the Plant Growth Facility for

531 careful maintenance of the Danforth Center phenotyping facilities. We thank Katie Liberatore

532 and Shahryar Kianian for images of wheat (Triticum aestivum L.). We would also like to thank

533 all of the other people who have given us input on the PlantCV project in person or on GitHub.

534

\section{REFERENCES}

536 Abbasi A., Fahlgren N. 2016. Naive Bayes pixel-level plant segmentation. In: 2016 IEEE

537 Western New York Image and Signal Processing Workshop (WNYISPW). 1-4. DOI:

$538 \quad 10.1109 /$ WNYIPW.2016.7904790.

539 Acosta-Gamboa LM., Liu S., Langley E., Campbell Z., Castro-Guerrero N., Mendoza-Cozatl D.,

540 Lorence A. 2017. Moderate to severe water limitation differentially affects the phenome and

541 ionome of Arabidopsis. Functional Plant Biology: FPB 44:94-106. DOI: 10.1071/FP16172.

542 Atkinson JA., Lobet G., Noll M., Meyer PE., Griffiths M., Wells DM. 2017. Combining semi-

543 automated image analysis techniques with machine learning algorithms to accelerate large 
$544 \quad$ scale genetic studies. GigaScience. DOI: 10.1093/gigascience/gix084.

545 Bookstein FL. 1991. Morphometric tools for landmark data Cambridge University Press. New 546 York.

547 Bookstein FL. 1997. Morphometric Tools for Landmark Data: Geometry and Biology.

$548 \quad$ Cambridge University Press.

549 Bradski G. 2000. The opencv library. Doctor Dobbs Journal 25:120-126.

550 Ćwiek-Kupczyńska H., Altmann T., Arend D., Arnaud E., Chen D., Cornut G., Fiorani F.,

551 Frohmberg W., Junker A., Klukas C., Lange M., Mazurek C., Nafissi A., Neveu P., van

552 Oeveren J., Pommier C., Poorter H., Rocca-Serra P., Sansone S-A., Scholz U., van Schriek

553 M., Seren Ü., Usadel B., Weise S., Kersey P., Krajewski P. 2016. Measures for

554 interoperability of phenotypic data: minimum information requirements and formatting.

555 Plant Methods 12:44. DOI: 10.1186/s13007-016-0144-4.

556 Dryden IL., Mardia KV. 2016. Statistical Shape Analysis: With Applications in R. John Wiley \& 557 Sons.

558 Duarte M. 2015. Notes on Scientific Computing for Biomechanics and Motor Control. GitHub

559 repository.

560 Dudley JT., Butte AJ. 2009. A quick guide for developing effective bioinformatics programming $561 \quad$ skills. PLoS Computational Biology 5:e1000589. DOI: 10.1371/journal.pcbi.1000589.

562 Fahlgren N., Feldman M., Gehan MA., Wilson MS., Shyu C., Bryant DW., Hill ST., McEntee

563 CJ., Warnasooriya SN., Kumar I., Ficor T., Turnipseed S., Gilbert KB., Brutnell TP.,

564 Carrington JC., Mockler TC., Baxter I. 2015. A versatile phenotyping system and analytics

565 platform reveals diverse temporal responses to water availability in Setaria. Molecular Plant 566 8:1520-1535. DOI: 10.1016/j.molp.2015.06.005. 
567 Fahlgren N., Gehan MA., Baxter I. 2015. Lights, camera, action: high-throughput plant

568 phenotyping is ready for a close-up. Current Opinion in Plant Biology 24:93-99. DOI:

$569 \quad$ 10.1016/j.pbi.2015.02.006.

570 Feldman MJ., Paul RE., Banan D., Barrett JF., Sebastian J., Yee M-C., Jiang H., Lipka AE.,

571 Brutnell TP., Dinneny JR., Leakey ADB., Baxter I. 2017. Time dependent genetic analysis

572 links field and controlled environment phenotypes in the model C4 grass Setaria. PLoS

573 Genetics 13:e1006841. DOI: 10.1371/journal.pgen.1006841.

574 Freeman H. 1961. On the encoding of arbitrary geometric configurations. IRE Transactions on

575 Electronic Computers EC-10:260-268. DOI: 10.1109/TEC.1961.5219197.

576 Furbank RT., Tester M. 2011. Phenomics--technologies to relieve the phenotyping bottleneck.

577 Trends in Plant Science 16:635-644. DOI: 10.1016/j.tplants.2011.09.005.

578 Gonzalez RC., Woods RE. 2002. Digital Image Processing. Prentice Hall.

579 Gunz P., Mitteroecker P. 2013. Semilandmarks: a method for quantifying curves and surfaces.

$580 \quad$ Hystrix, the Italian Journal of Mammalogy 24:103-109.

581 Gunz P., Mitteroecker P., Bookstein FL. 2005. Semilandmarks in Three Dimensions. In: Slice

582 DE ed. Modern Morphometrics in Physical Anthropology. Developments in Primatology:

583 Progress and Prospects. Springer US, 73-98. DOI: 10.1007/0-387-27614-9_3.

584 Hunter JD. 2007. Matplotlib: A 2D graphics environment. Computing in Science \& Engineering 585 9:90-95. DOI: 10.1109/MCSE.2007.55.

586 Jones E., Oliphant T., Peterson P. 2014. SciPy: Open source scientific tools for Python.

587 Kaehler A., Bradski G. 2016. Learning OpenCV 3: Computer Vision in C++ with the OpenCV 588 Library. O'Reilly Media, Inc.

589 Kluyver T., Ragan-Kelley B., Pérez F., Granger B., Bussonnier M., Frederic J., Kelley K., 
Hamrick J., Grout J., Corlay S., Ivanov P., Avila D., Abdalla S., Willing C., Jupyter

Development Team. 2016. Jupyter Notebooks - a publishing format for reproducible

592

computational workflows. In: Loizides F, Schmidt B eds. Positioning and Power in

593

Academic Publishing: Players, Agents and Agendas: Proceedings of the 20th International

594

Conference on Electronic Publishing. Amsterdam: IOS Press, 87-90. DOI: 10.3233/978-1-

595 61499-649-1-87.

Liberti L., Lavor C., Maculan N., Mucherino A. 2014. Euclidean distance geometry and applications. SIAM Review 56:3-69. DOI: 10.1137/120875909.

Lobet G. 2017. Image analysis in plant sciences: Publish then perish. Trends in Plant Science. DOI: $10.1016 / \mathrm{j} . t p l a n t s .2017 .05 .002$.

Lobet G., Draye X., Périlleux C. 2013. An online database for plant image analysis software

601 tools. Plant Methods 9:38. DOI: 10.1186/1746-4811-9-38.

Mangalam H. 2002. The Bio* toolkits--a brief overview. Briefings in Bioinformatics 3:296-302.

603 DOI: $10.1093 / \mathrm{bib} / 3.3 .296$.

McKinney W., Others. 2010. Data structures for statistical computing in python. In: Proceedings 605 of the 9th Python in Science Conference. SciPy Austin, TX, 51-56.

606

607

Millman KJ., Aivazis M. 2011. Python for scientists and engineers. Computing in Science \&

Engineering 13:9-12. DOI: 10.1109/MCSE.2011.36.

608 Minervini M., Abdelsamea MM., Tsaftaris SA. 2014. Image-based plant phenotyping with

609 incremental learning and active contours. Ecological Informatics 23:35-48. DOI:

$610 \quad$ 10.1016/j.ecoinf.2013.07.004.

611 Oliphant TE. 2007. Python for scientific computing. Computing in Science \& Engineering 9:10612 20. DOI: 10.1109/MCSE.2007.58. 
613 Otsu N. 1979. A threshold selection method from gray-level histograms. IEEE Transactions on $614 \quad$ Systems, Man, and Cybernetics 9:62-66.

615 Pauli D., Chapman SC., Bart R., Topp CN., Lawrence-Dill CJ., Poland J., Gore MA. 2016. The 616 quest for understanding phenotypic variation via integrated approaches in the field 617 environment. Plant Physiology 172:622-634. DOI: 10.1104/pp.16.00592.

618 Perez-Riverol Y., Gatto L., Wang R., Sachsenberg T., Uszkoreit J., Leprevost F da V., Fufezan 619 C., Ternent T., Eglen SJ., Katz DS., Pollard TJ., Konovalov A., Flight RM., Blin K., 620 Vizcaíno JA. 2016. Ten simple rules for taking advantage of Git and GitHub. PLoS 621 Computational Biology 12:e1004947. DOI: 10.1371/journal.pcbi.1004947.

622 Pound MP., Atkinson JA., Townsend AJ., Wilson MH., Griffiths M., Jackson AS., Bulat A., 623 Tzimiropoulos G., Wells DM., Murchie EH., Pridmore TP., French AP. 2017. Deep machine 624 learning provides state-of-the-art performance in image-based plant phenotyping. 625 GigaScience. DOI: 10.1093/gigascience/gix083.

626 R Core Team. 2017. R: A Language and Environment for Statistical Computing.

627 RStudio Team. 2016. RStudio: Integrated Development Environment for R. Boston, MA:

628 RStudio, Inc.

629 Scharr H., Minervini M., French AP., Klukas C., Kramer DM., Liu X., Luengo I., Pape J-M., 630 Polder G., Vukadinovic D., Yin X., Tsaftaris SA. 2016. Leaf segmentation in plant 631 phenotyping: a collation study. Machine Vision and Applications 27:585-606. DOI: $632 \quad 10.1007 / \mathrm{s} 00138-015-0737-3$.

633 Schlager S., Jefferis G., Schlager MS. 2016. Package "Morpho."

634 Schneider CA., Rasband WS., Eliceiri KW. 2012. NIH Image to ImageJ: 25 years of image 635 analysis. Nature Methods 9:671-675. 
636 Singh A., Ganapathysubramanian B., Singh AK., Sarkar S. 2016. Machine learning for high637 throughput stress phenotyping in plants. Trends in Plant Science 21:110-124. DOI:

$638 \quad$ 10.1016/j.tplants.2015.10.015.

639 Tovar J., Hoyer JS., Lin A., Tielking A., Callen S., Castillo E., Miller M., Tessman M., Fahlgren 640 N., Carrington J., Nusinow D., Gehan MA. 2017. Raspberry Pi powered imaging for plant 641 phenotyping. bioRxiv:183822. DOI: 10.1101/183822.

642 Ubbens JR., Stavness I. 2017. Deep Plant Phenomics: A deep learning platform for complex 643 plant phenotyping tasks. Frontiers in Plant Science 8:1190. DOI: 10.3389/fpls.2017.01190. 644 van der Walt S., Colbert SC., Varoquaux G. 2011. The NumPy array: A structure for efficient 645 numerical computation. Computing in Science \& Engineering 13:22-30. DOI:

$646 \quad$ 10.1109/MCSE.2011.37.

647 van der Walt S., Schönberger JL., Nunez-Iglesias J., Boulogne F., Warner JD., Yager N., 648 Gouillart E., Yu T., scikit-image contributors. 2014. scikit-image: image processing in 649 Python. PeerJ 2:e453. DOI: 10.7717/peerj.453.

650 Wickham H. 2009. ggplot2: Elegant Graphics for Data Analysis. Springer New York.

651 Wilson G., Aruliah DA., Brown CT., Chue Hong NP., Davis M., Guy RT., Haddock SHD., Huff 652 KD., Mitchell IM., Plumbley MD., Waugh B., White EP., Wilson P. 2014. Best practices for 653 scientific computing. PLoS Biology 12:e1001745. DOI: 10.1371/journal.pbio.1001745.

654 Zack GW., Rogers WE., Latp SA. 1977. Automatic measurement of sister chromatid exchange 655 frequency. Journal of Histochemistry and Cytochemistry 25:741-753. 
Figure 1 (on next page)

Diagram of the components of PlantCV.

(A) PlantCV is an open-source, open-development suite of image analysis tools. PlantCV contains a library of modular Python functions that can be assembled into simple sequential or branching/merging processing pipelines. Image processing pipelines, which process single images (possibly containing multiple plants), can be deployed over large image sets using PlantCV parallelization, which outputs an SQLite database of both measurements and image/experimental metadata. (B) Overview of the structure of the SQLite database. 


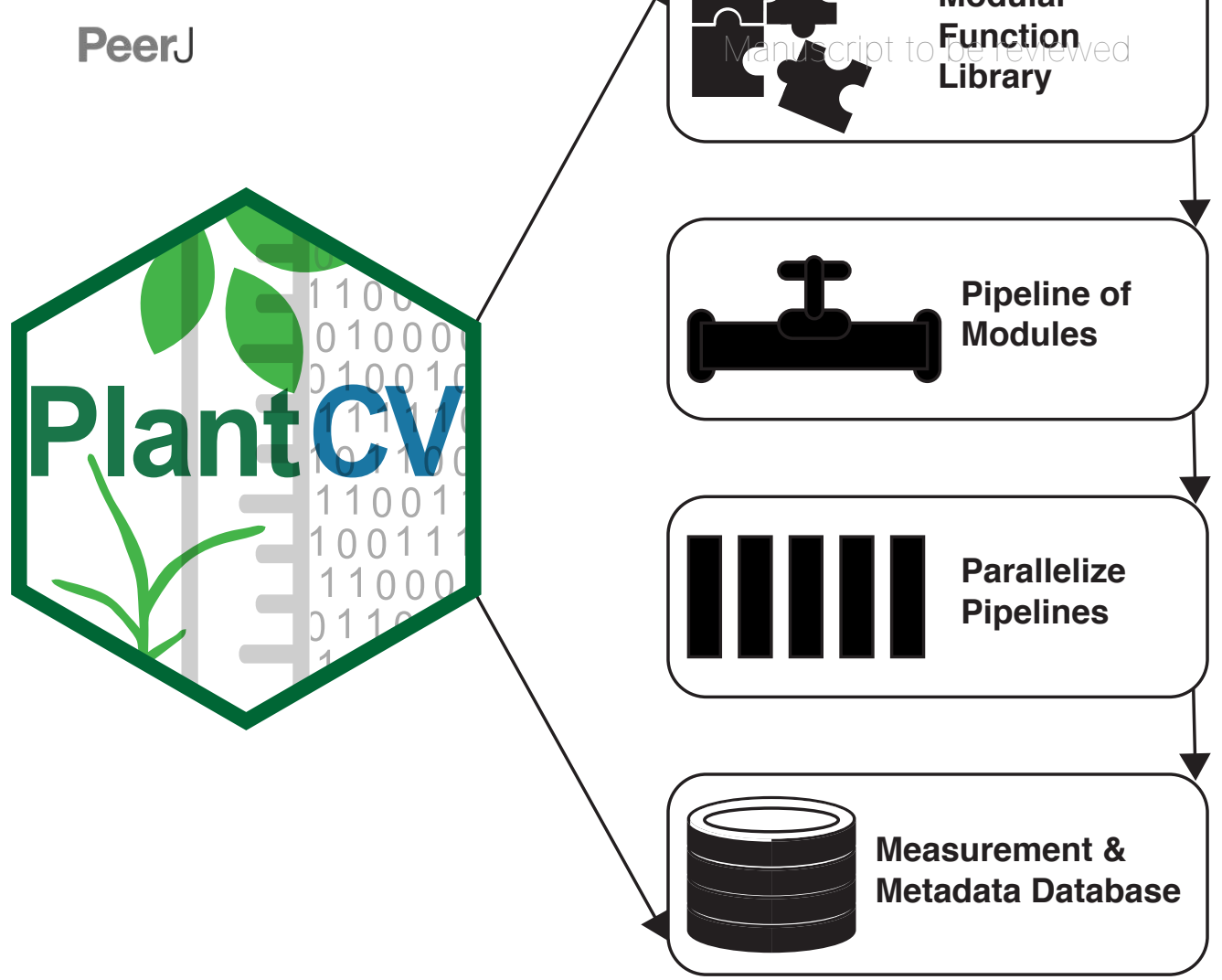

B

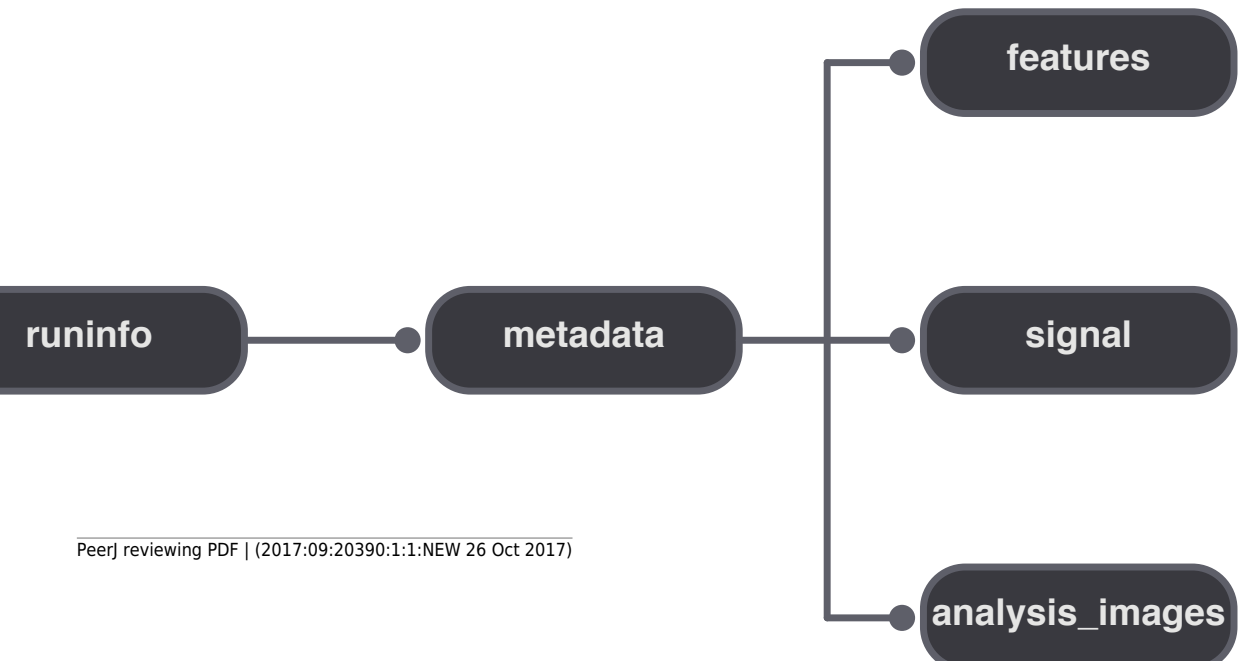




\section{Figure 2 (on next page)}

Analysis of images containing multiple plants.

New functions have been added to PlantCV $v 2$ that enable individual plants from images containing multiple plants to be analyzed. The 'cluster_contours' function clusters contour objects using a flexible grid arrangement (approximate rows and columns defined by a user). (A) An image produced by 'cluster_contours' in debug mode highlights plants by their cluster group with unique colors on a sequential scale. The 'cluster_contours_split_img' function creates a new image for each cluster group. The resulting images of individual plants can be processed by standard PlantCV methods. (B) The 'cluster_contours_split_img' function was used to split the full image into individual plants. The shape of each plant was then analyzed with 'analyze_objects' and printed on a common image background. 
Figure 3 (on next page)

Leaf segmentation by a distance-based watershed transformation.

The watershed segmentation function can be used to segment and estimate the number of objects in an image. For the three example images, the watershed segmentation function was used to estimate the number of leaves for Arabidopsis thaliana (estimated leaf count for top: 13, middle: 14, and bottom: 8). Images shown are the output from the 'watershed_segmentation' function ( $A, C, E)$ and the segmented plants (B, D, F). 


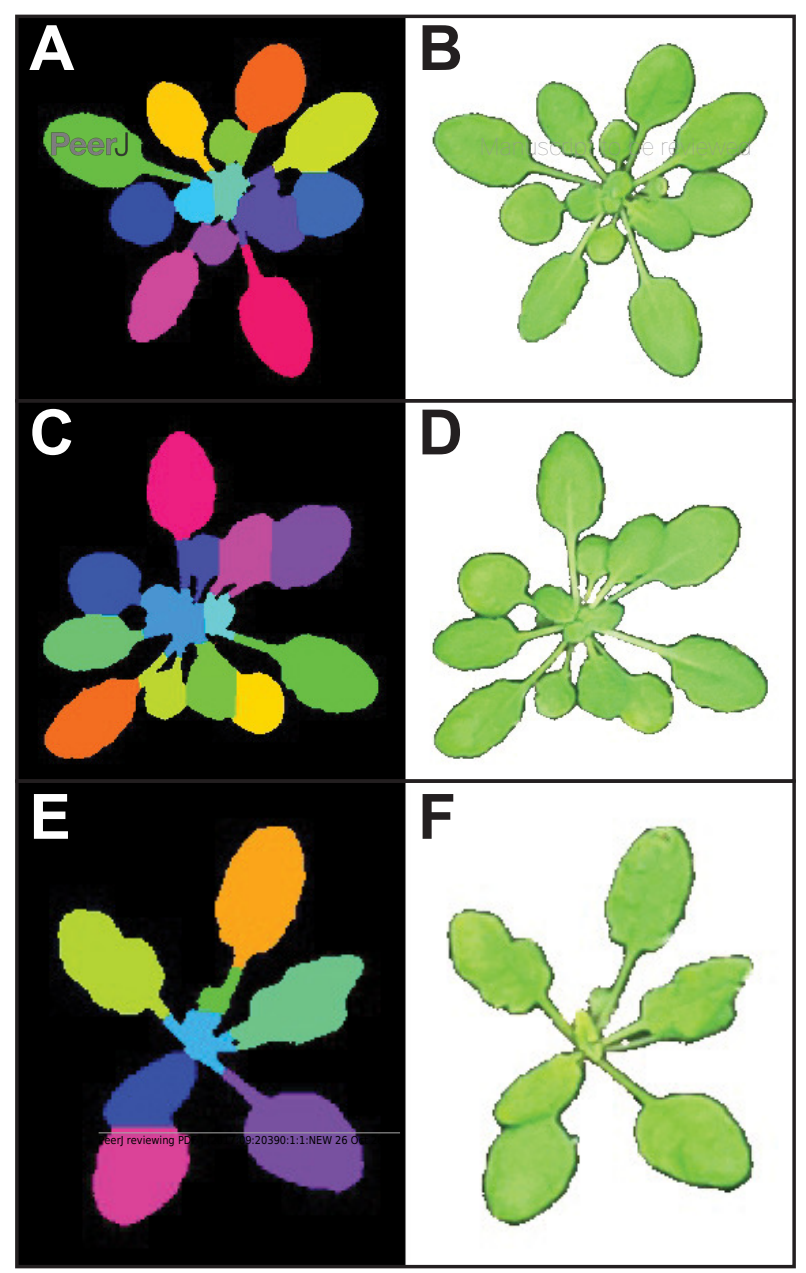




\section{Figure 4 (on next page)}

Landmark-based analysis of plant shape in PlantCV.

(A) Automatic identification of leaf tip landmarks using the 'acute' and 'acute_vertex' functions (blue dots). (B) Geometrically homologous semi/pseudo-landmarks across both the $x$ - and $y$-axes. Semi/pseudo-landmarks identified by scanning the $x$-axis are denoted by light blue (top side of the contour), brown (bottom side of the contour), and light orange (centroid location of horizontal bins) dots. Semi/pseudo-landmarks identified by scanning the $y$-axis are denoted by dark blue (left side of the contour), pink (right side of the contour), and dark orange (centroid location of vertical bins) dots. The plant centroid is plotted larger in red. (C) A representation of the rescaled plant landmarks identified in panel (A). White points correspond to the leaf tips. The orange point is the location of the plant centroid. The blue point is the location of the plant centroid where the plant emerges from the soil. Red lines are the vertical distance from leaf tip points relative to the plant centroid. (D) Analysis of the average scaled vertical distance from each leaf tip to the centroid diverges in response to water limitation. 

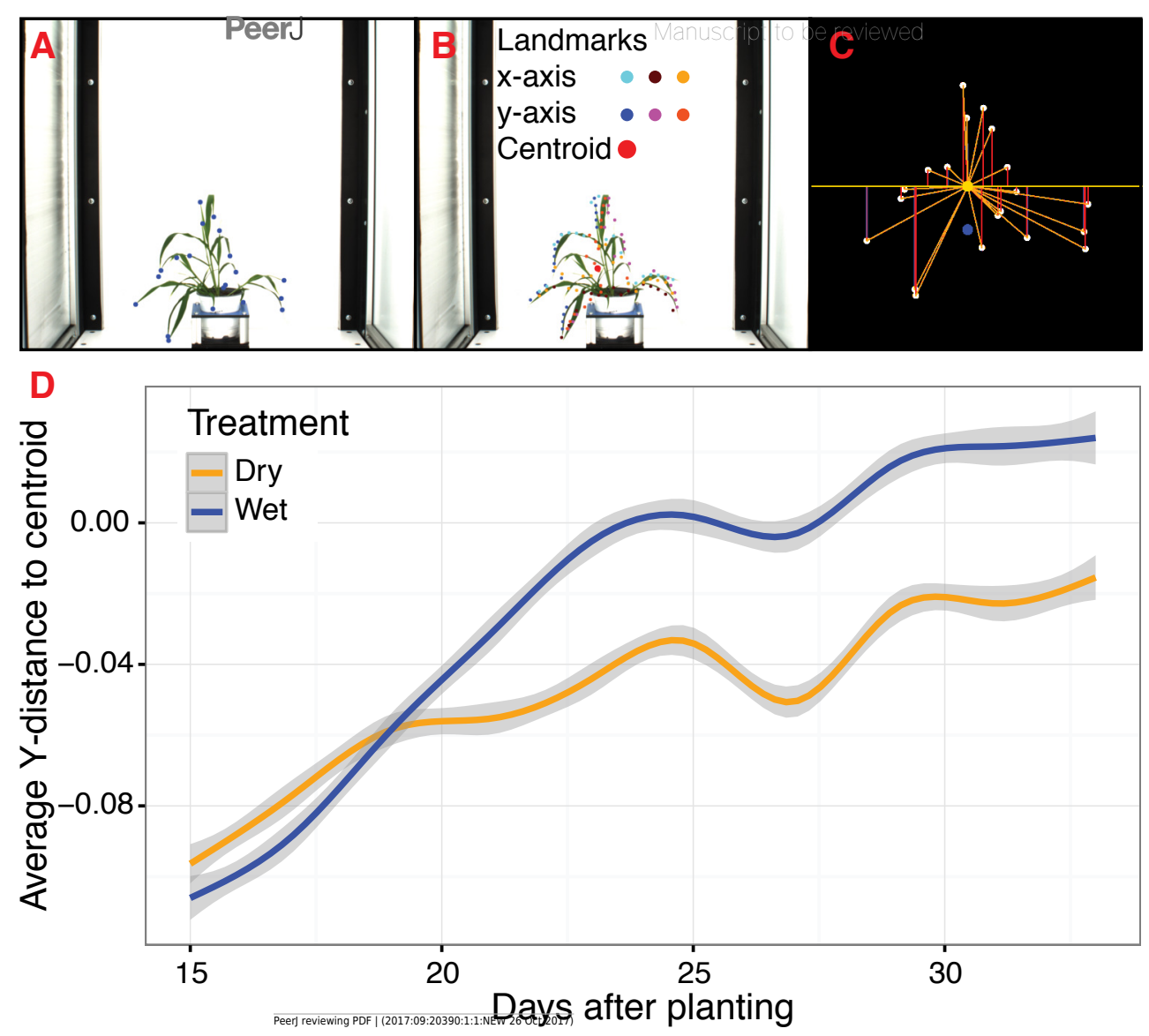
Figure $\mathbf{5}$ (on next page)

Plant segmentation using a naive Bayes classifier.

Correlation between plant area in pixels ( $\mathrm{px}$ ) detected using thresholding pipelines (Fahlgren et al., 2015) on the $x$-axis compared to plant area detected using a trained naive Bayes classifier on the $y$-axis. (A) Side-view images. (B) Top-view images. 

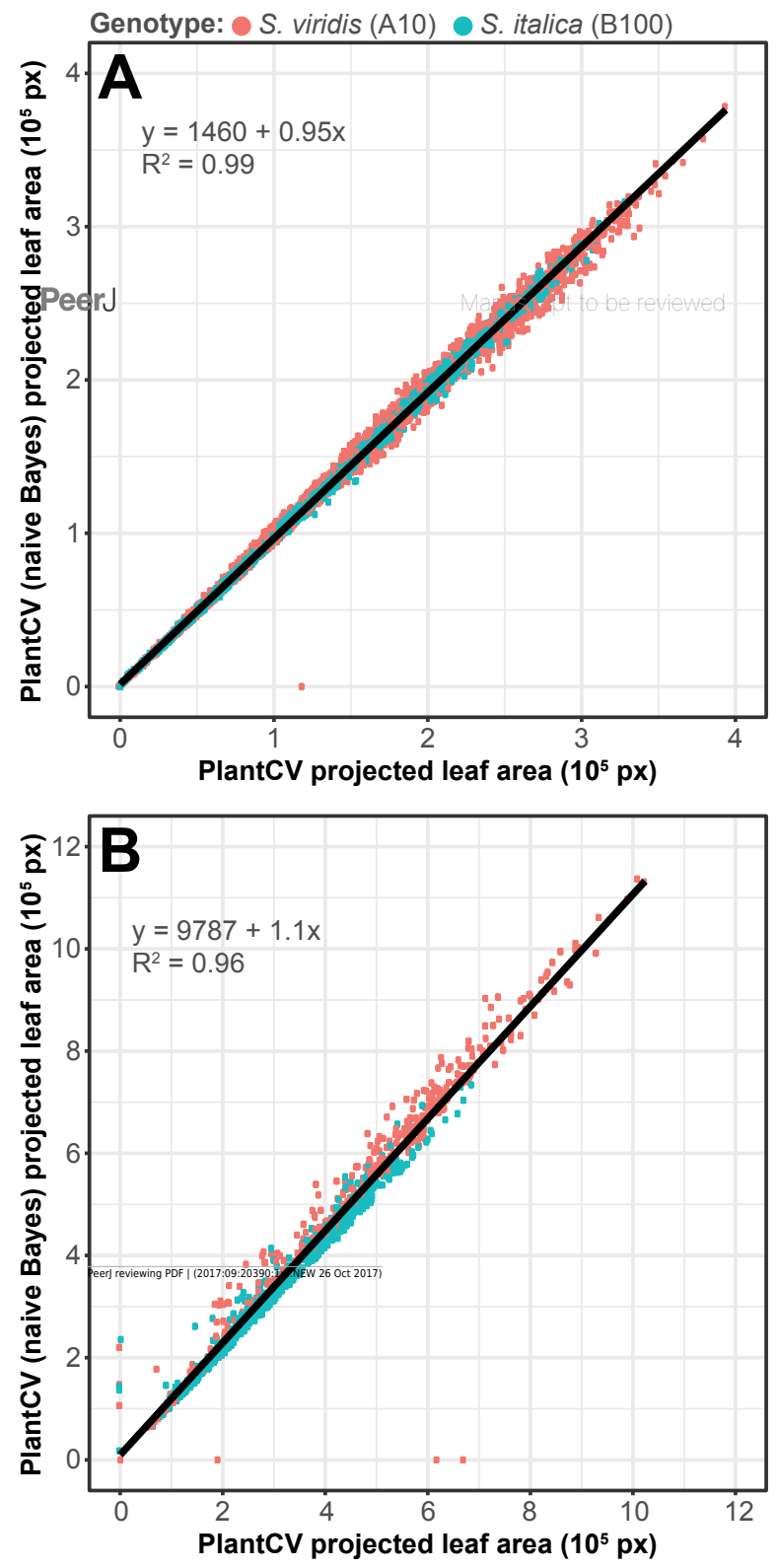


\section{Figure 6 (on next page)}

Simultaneous segmentation of four feature groups using the naive Bayes classifier.

An example of the naive Bayes classifier used to assign pixels into 4 classes: background, unaffected plant tissue, chlorotic tissue, and wheat stem rust pustules. (A) Probability density functions (PDFs) from the 'plantcv-train.py' script that show hue, saturation, and value color channel distributions of four classes estimated from training data. (B) Example of a classified image. Photo credit: Katie Liberatore and Shahryar Kianian. (C) Example of a merged pseudocolored image with pixels classified by the 'naive_bayes_classifier' as background (black), unaffected leaf tissue (green), chlorotic leaf tissue (blue), and pustules (red). 


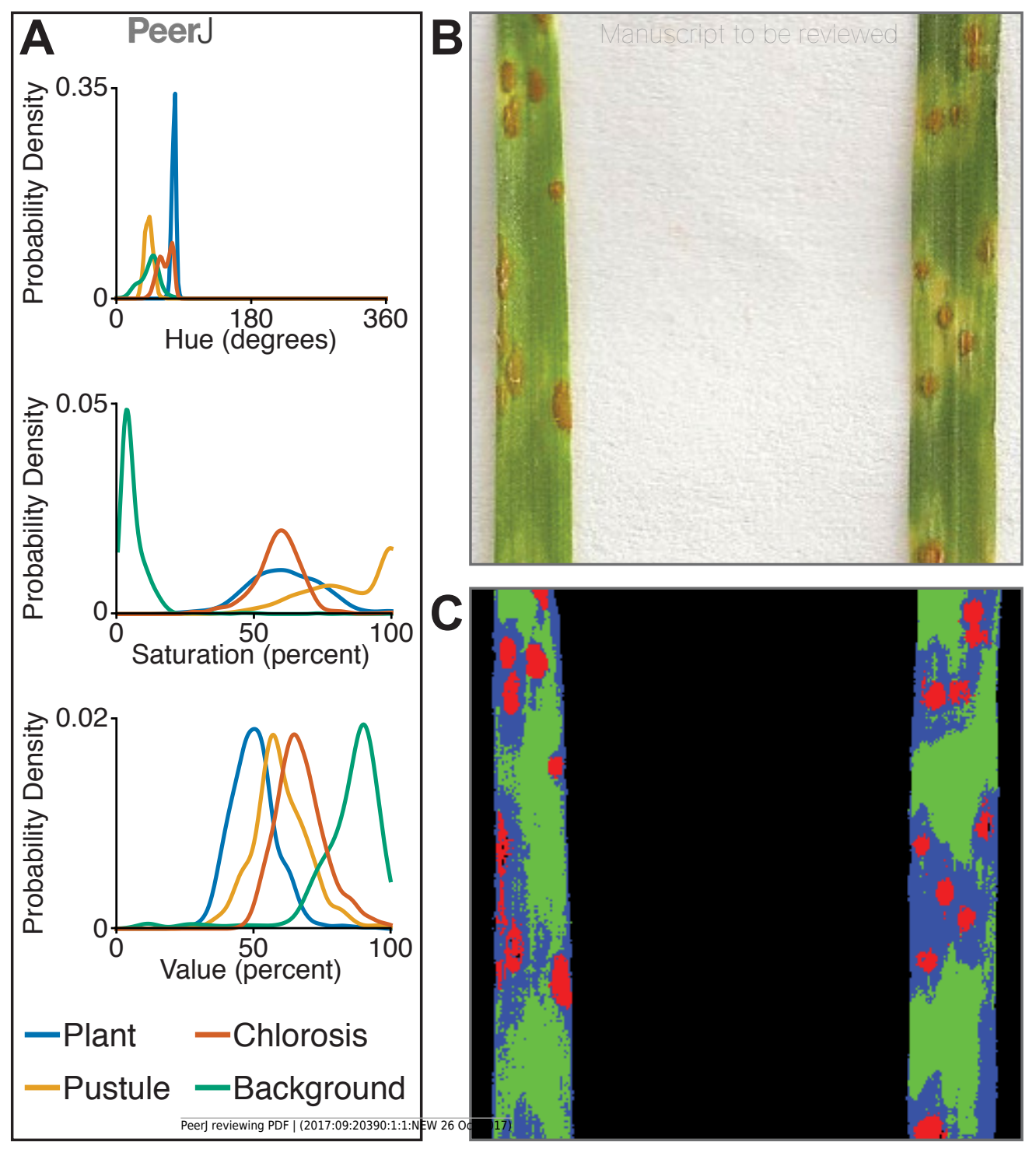

